\title{
L'aptitude des mycorhizes à protéger les plantes contre les maladies : panacée ou chimère?
}

\author{
R. PERRIN \\ I.N.R.A., Station de Recherches sur la Flore pathogène dans le Sol \\ 17, rue Sully, 21034 Dijon Cedex
}

\begin{abstract}
Résumé
L'auteur s'interroge sur l'aptitude des mycorhizes à protéger les plantes contre les maladies. Des exemples extraits de la littérature ou des expériences de l'auteur, révèlent que l'association mycorhizienne peut être source d'amélioration, mais également d'aggravation de l'état sanitaire des plantes.

L'effet protecteur n'a, à ce jour, été démontré que pour les maladies d'origine tellurique. L'expression de ce potentiel naturel demeure subordonnée à de nombreux facteurs : nature de l'hôte, du champignon mycorhizogène, pathogène et des conditions de l'environnement souterrain.

L'exploitation pratique de ce phénomène, parfois très efficace, nécessite de combler les lacunes de notre connaissance des conditions favorables à l'expression de l'aptitude prophylactique propre à certaines associations symbiotiques.
\end{abstract}

\section{Introduction}

Depuis l'apparition au grand jour de la notion de champignon associé aux racines (Frank, 1885), les nombreux travaux consacrés aux mycorhizes ont révélé leur importance dans le domaine végétal. Au cours des dix dernières années les recherches sur les symbioses ont connu un large essor, démontrant que l'association mycorhizienne est un phénomène universel, indispensable à la plante. Et pourtant combien d'études physiologiques, de programme d'amélioration génétique... ont oublié, et continuent d'ignorer les mycorhizes pourtant partie intégrante de la plante (Graninazzi et al., 1982).

On prête aux mycorhizes de multiples vertus, maintes fois mises en évidence : amélioration de la surface absorbante du système racinaire, de la sélectivité de l'absorption, de l'accumulation, de la solubilisation de certains éléments nutritifs, de la longévité de la racine; augmentation de la tolérance aux toxines, de la résistance aux conditions adverses (sécheresse, salinité...) ; protection contre les agents pathogènes. Cette capacité à s'opposer aux agents pathogènes a suscité bien peu d'intérêt, en regard de son énorme potentiel, et de l'impact de certaines maladies sur le ren- 
dement des cultures. Les mycorhizes ont été totalement délaissées dans les stratégies actuelles de lutte contre les maladies. D'ailleurs cette aptitude de protection est encore très controversée. Certains voient dans les mycorhizes une solution universelle et radicale aux problèmes de maladie de toutes origines, d'autres alu contraire, les jugent d'emblée inopérantes. Où est la vérité ? Les mycorhizes constituent-elles de précieux alliés ou un mirage dans la lutte contre les agents pathogènes?

\section{La protection contre les maladies : une réalité bien établie, mais non une règle générale}

La grande majorité des publications traitant de la protection des plantes contre les maladies par les mycorhizes attestent d'un effet bénéfique. Rares sont les travaux qui font état d'une aggravation de la maladie ou de l'absence d'influence des mycorhizes. Cette disproportion pourrait laisser croire au remède miracle si toutes les tentatives soldées par un échec avaient donné lieu à publication ! II est possible à travers quelques exemples de mieux cerner la réalité.

Phytophthora cinnamomi compte parmi les agents pathogènes les plus redoutés à travers le monde. $\mathrm{Il}$ est à l'origine de maladies racinaires conduisant au dépérissement de nombreuses espèces feuillues et résineuses. Il est particulièrement dommageable au Chamaecyparis lawsoniana. BARTSCH et al. (1981) ont étudié l'influence de l'association endomycorhizienne sur la sensibilité de cette espèce cultivée en conteneur.

Cette étude est source de plusieurs enseignements. La gravité et le cours de la maladie ne sont pas modifiés lorsque le champignon mycorhizogène est introduit dans le substrat d'élevage en même temps que l'agent pathogène. Par contre, les plantes échappent à la maladie si le champignon mycorhizogène précède de six mois l'introduction du parasite. La protection contre le Phytophthora cinnamomi ne s'exerce donc que si la mycorhize est bien établie (fig. 1). De plus, l'association mycorhizienne réalisée à partir d'une seule souche de Glomus mosseae n'exerce qu'une influence limitée à un simple délai dans l'apparition des symptômes mais qui n'empêche pas la mort des plantes. Au contraire la protection est durable lorsque les Chamaecyparis ont été confrontés à une population mélangée naturelle. Le bénéfice révélé ici peut découler d’une meilleure association mycorhizienne assurée par plusieurs espèces, passant éventuellement par des effets synergiques, ou d'une meilleure efficacité de certaines espèces de la population naturelle. Si cette étude démontre parfaitement que l'association mycorhizienne confère au Chamaecyparis une protection contre $P$. cinnamomi, elle indique que cette protection demeure subordonnéc à l'installation préalable du ou des champignons mycorhizogènes, et dépend de la nature du symbiote. D'autres études constatent un effet positif de l'association endomycorhizienne de diverses plantes sur l'état sanitaire de leur système racinaire (Woodhrab) et al., 1977...).

Toutefois, l'association mycorhizienne est parfois source d'aggravation des maladies racinaires. Ainsi Ross (1972) observe que l'association mycorhizienne entre un cultivar de soja (D60-Glycine max) et une espèce de l'ex-genre Endogone conduit à une aggravation de la pourriture racinaire due à Phytophtora megasperma var. sojae (tabl. 1). Cela se traduit par une fréquence plus élevée de plantes atteintes en 


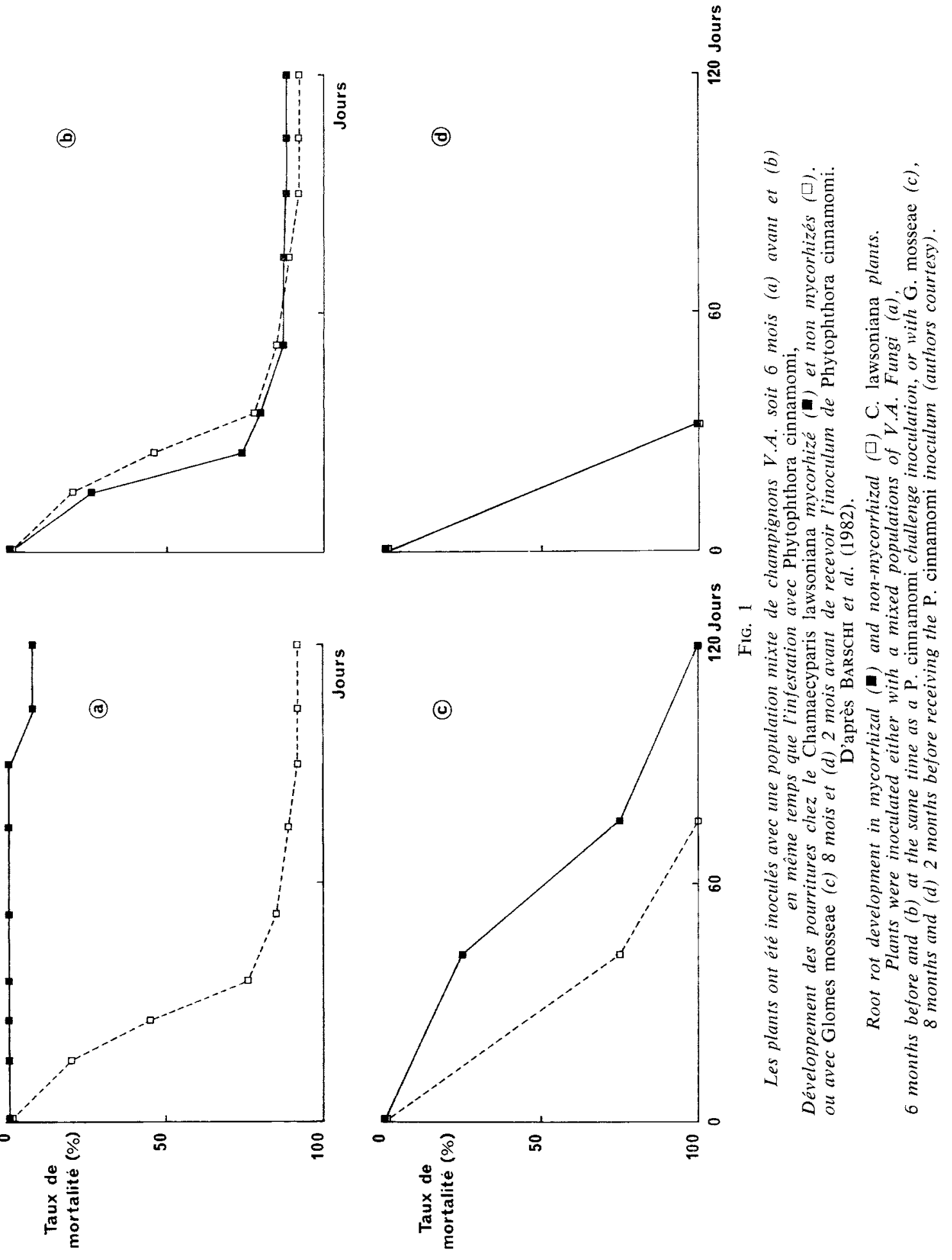


présence d'Endogone (21) qu'en son absence (9). La même espèce mycorhizogène associée à un autre cultivar de Soja (Lee) manifestement moins sensible, n'influence pas le cours de la maladie et améliore sensiblement le rendement. Ce résultat met en lumière une particularité fondamentale : la protection contre certaines maladies est une aptitude propre à la mycorhize. C'est l'expression d'une entité génétique bien déterminée définic par l'interaction entre le génome de la plante et celui du champignon.

\section{TABleau 1}

Influence de la nature de l'hôte sur les relations entre endomycorhizes et Phytophthora megasperma, agent de pourriture des racines du Soja.

D'après Ross (1972).

Influence of host plant on the relationships between endomycorrhizae and Phytophthora megasperma, cause of root rot of soybean.

\begin{tabular}{|c|c|c|c|c|}
\hline Cultivar & Infection & $\begin{array}{l}\text { Nombres de } \\
\text { plantes mortes } \\
\text { (a) }\end{array}$ & $\begin{array}{c}\text { Nombres de } \\
\text { plantes attcintes }\end{array}$ & $\begin{array}{l}\text { Poids moyen } \\
\text { par plante }(\mathrm{g})\end{array}$ \\
\hline Lee & $\begin{array}{l}\text { Phytophtora } m \text {. } \\
\text { Phytophtora } \\
\quad+\text { Endogone sp. } \\
\text { Témoin }\end{array}$ & $\begin{array}{l}0 \\
0 \\
0\end{array}$ & $\begin{array}{l}5 \\
5 \\
0\end{array}$ & $\begin{array}{l}153 \\
204 \\
181\end{array}$ \\
\hline D 60 & $\begin{array}{l}\text { Phytophtora m. } \\
\text { Phytophtora } \\
\quad+\text { Endogone sp. } \\
\text { Témoin }\end{array}$ & $\begin{array}{l}0 \\
8 \\
0\end{array}$ & $\begin{array}{c}9 \\
21 \text { (b) } \\
0\end{array}$ & $\begin{array}{l}126 \\
110 \\
284\end{array}$ \\
\hline
\end{tabular}

(a) 24 plantes par traitement.

24 plants for each treatiment.

(b) $\mathrm{Y}$ compris les plantes mortes. Dead plants included.

La protection des plantes n'est pas l'apanage des endomycorhizes. En effet, certaines espèces d'ectomycorhizes associées à des espèces du genre Pinus offrent une bonne protection contre les attaques de P. cinnamomi (Marx, 1969) (tabl. 2). Les racines de Pinus taeda mycorhizées par Laccaria laccata, Leucopaxillus cerealis var piceana, Suillus luteus échappent à l'infection par $P$. cinnamomi, tandis que $P$. tinctorius qui ne forme qu'un manchon mycélien (manteau) incomplet, ne protège que 81 p. 100 des racines mycorhizées. Toutes les pointes racinaires latérales sont infectées par le parasite, ainsi que les racines courtes voisines des racines mycorhizées.

Toutefois 75 p. 100 de ces racines restent indemnes lorsque les racines mycorhizées à proximité sont associées à Leucopaxillus cerealis. La proportion est moindre, 23 p. 100, lorsque le symbiote est Suillus luteus. Ces deux types de mycorhizes paraissent aptes à une protection à distance, au contraire de celles formées avec Pisolithus tinctorius qui exerceraient un simple effet de barrière, pouvant être mis en défaut dès lors qu'elle est discontinue. 
TABLEAU 2

Influence de l'association mycorhizienne sur les pourritures racinaires produites par Phytophthora cinnamomi chez Pinus taeda.

D'après Marx (1969).

Influence of mycorrhization on Phytophthora cinnamomi root rot of Pinus taeda.

\begin{tabular}{|c|c|c|c|c|c|c|}
\hline \multirow{2}{*}{$\begin{array}{l}\text { Champignon } \\
\text { mycorhizogène }\end{array}$} & \multicolumn{2}{|c|}{ Mycorhizes } & \multicolumn{2}{|c|}{$\begin{array}{c}\text { Racines courtes } \\
\text { adjacentes }\end{array}$} & \multicolumn{2}{|c|}{$\begin{array}{c}\text { Pointes racinaires } \\
\text { latérales }\end{array}$} \\
\hline & $\begin{array}{c}\mathrm{Nb} \\
\text { inocules : }\end{array}$ & $\begin{array}{c}\% \\
\text { infection : }\end{array}$ & $\begin{array}{c}\mathrm{Nb} \\
\text { inoculés }\end{array}$ & $\begin{array}{c}\% \\
\text { infection * }\end{array}$ & $\begin{array}{c}\mathrm{Nb} \\
\text { inoculés }\end{array}$ & infection : \\
\hline Laccaria laccata . . & 7 & 0 & 29 & 100 & 8 & 100 \\
\hline $\begin{array}{c}\text { Leucopaxillus cerealis } \\
\text { var. piceana } \ldots . .\end{array}$ & 27 & 0 & 32 & 25 & 6 & 100 \\
\hline Pisolithus tinctorius & 42 & 19 & 13 & 100 & 6 & 100 \\
\hline Suillus luteus & 9 & 0 & 34 & 77 & 6 & 100 \\
\hline Témoin non mycorhizé & - & - & 23 & 100 & 8 & 100 \\
\hline
\end{tabular}

$\therefore$ Avec P. cinnamomi.

With P. cinnanomi.

D'autres maladies d'origine tellurique voient leur gravité atténuée sous l'effet des mycorhizes. Ainsi le Laccaria laccata dont les carpophores sont fréquents dans nos forêts, offre aux résineux une protection efficace contre le redoutable agent de nécrose racinaire et de fonte des semis : Fusarium oxysporum Schlecht (SINCLAIR et al., 1975). Ce symbiote serait d'ailleurs le seul capable de s'opposer dans le sol à un agent pathogène avant même la formation de mycorhize.

Perrin \& Garbaye (1982) ont montré que l'association mycorhizienne réalisée entre Hebeloma crustuliniforme et le hêtre (Fagus sylvatica) maintient à un niveau très bas le potentiel infectieux ( $\left.{ }^{*}\right)$ d'un sol ou d'un substrat infesté par Pythium spp.

Corte (1969) observe que les mycorhizes formées par Suillus granulatus offrent à Pinus excelsa une protection vis-à-vis de la pourriture racinaire engendrée par Rhizoctonia spp.

Les études consacrées à l'effet protecteur de l'association mycorhizienne des plantes vis-à-vis des agents pathogènes telluriques montrent qu'il est possible de trouver des symbiotes efficaces contre la plupart des agents pathogènes du sol, en particulier vis-à-vis des parasites fongiques les plus dommageables aux essences forestières.

Les relations entre nématodes phytophages et mycorhizes sont connues à travers une littérature tout aussi copieuse. L'association endomycorhizienne de différentes plantes comme le tabac, la tomate, l'avoine avec Glomus mosseae conduit à une

(*) Aptitude d'un sol à produire une maladie sur une population d'hôtes sensibles. 
atténuation des effets maladifs produits par Meloidogyne incognila, espèce également parasite de plantes pérennes (SIKORA \& SCHONBECK, 1975). Une aggravation des dégâts est rapportée par ATILANo et al. (1976) à la suite des attaques de Meloidogyne arenaria sur la vigne associéc à diverses espèces d'endogonacées. Bien que les témoignages faisant état d'une amélioration de l'état sanitaire de la plante représentent l'essentiel des publications, l'association mycorhizienne peut aboutir comme pour les champignons parasites, à un accroissement des effets maladifs.

\subsection{Mycorhization et maladies cuériennes}

Plus rares sont les travaux consacrés à l'influence de l'association mycorhizienne sur les maladies aériennes ou les maladies à virus. Tous ces travaux relatifs à des champignons endomycorbiziens à vésicule et arbuscule font état d'une aggravation de la maladie causée par des organismes tels que des rouilles sur haricot ou Botrytis cinerea sur laitue, ou encore différents virus sur des plantes variées (ScHonbeck, 1980). De ces connaissances très fragmentaires il semble ressortir que l'association endomycorhizienne aboutit à une sensibilisation des plantes à des parasites facultatifs ou obligatoires. Nos connaissances demeurent trop insuffisantes pour proposer des explications à ce phénomène. La symbiose n'affecte pas la résistance génétique de la plante (Denne, 1982). Toutefois les modifications du métabolisme de la plante mycorhizée peuvent avoir d'importants retentissements sur les mécanismes physiologiques à l'origine du processus de défense de la plante. On sait limportance de l'équilibre dans la nutrition des plantes et son influence sur la sensibilité des plantes aux parasites aériens; les excès de phosphore en particulier, ou d'autres éléments peuvent contribuer à amoindrir la résistance des parties aériennes à différentes maladies. L'amélioration de la nutrition de la plante est spécialement évoquée pour expliquer l'aggravation des maladies à virus.

Ce rapide tour d'horizon montre que des preuves incontestables ont été accumulées en faveur de l'existence de la protection des plantes contre les maladies grâce à la symbiose mycorhizienne. Nos connaissances actuelles indiquent que l'effet, lorsqu'il existe, est limité au site de l'interaction hôte-symbiote el n'est donc réalisé que pour les agents pathogènes telluriques. L'association mycorhizienne n'assure généralement pas une protection totale mais contribue à une nette réduction de la gravité des dommages. Cette particularité peut être simplement illustrée par l'étude de Marais \& Kotze (1976) (tabl. 3). On retrouve un trait caractéristique des phénomènes tampons naturels correspondant à des équilibres au sein desquels l'agent pathogène n'est pas exclu, mais où son activité, son impact sont considérablement limités. Il est illusoire d'attendre de la symbiose mycorhizienne une éradication totale des maladies, mais plutôt une substantielle atténuation caractérisée par une stabilité à la fois temporelle et vis-à-vis des conditions de l'environnement.

En forêt comme en pépinière, c'est l'absence de mycorhyzes qui constitue l'exception ou qui caractérise des systèmes culturaux très spécialisés. Par conséquent c'est plus la variabilité du bagage mycorhizien, donc la nature des associés, qui déterminera le comportement de la plante, notamment vis-à-vis des maladies d'origine tellurique. Or nous savons que les différences existant d'une espèce à l'autre peuvent être considérables. De plus l'effet de protection apparaît comme un potentiel qui s'exerce sous l'étroite dépendance de la nature de la plante, de l'associé fongique, de l'agent pathogène, mais également des conditions de l'environnement tellurique. 
TABLEAU 3

Influence de I'association mycorhyzienne de Pinus patula sur la maladie produite par Phytophthora cinnamomi.

D'après MARAIS \& KoTZE (1976).

Influence of mycorrhization on disease caused by Phytophthora cinnamomi on Pinus patula.

\begin{tabular}{|c|c|c|}
\hline Champignons mycorhizogènes & Après 3 semaines & Après 12 semaines \\
\hline $\begin{array}{l}\text { A manita muscaria } \\
\text { Boletus edulis }\end{array}$ & - & $\begin{array}{l}\text { Mortalité } \\
20 \%\end{array}$ \\
\hline Pas de champignons mycorhizogènes & $\begin{array}{l}\text { Premiers symptônes } \\
\text { (flétrissement) }\end{array}$ & $\begin{array}{l}\text { Mortalité } \\
80 \%\end{array}$ \\
\hline
\end{tabular}

2. Les conditions de l'expression du phénomène de protection contre les agents pathogènes

\subsection{Influence de la nature de l'hôte}

Les travaux de Ross (1972) fournissent un remarquable exemple de l'influence de la nature de l'hôte sur l'expression du phénomène de protection (tabl. 1). Selon le cultivar de soja utilisé, l'association avec la même espèce mycorhizogène conduit à des résultats opposés. Nous avons obtenu des résultats analogues avec des plants feuillus ectomycorhizés avec Hebeloma crustuliniforme. Associé au hêtre $H$. crustuliniforme entraîne une diminution du potentiel infectieux d'un sol infesté par Pythium spp., tandis qu'avec le chêne le potentiel infectieux n'est pas modifié. Une différence sensible existe entre les souches de la même uspèce (PERRIN \& Garbaye, 1982).

\subsection{Spécificité de l'aptitude prophylactique}

Quelques études, malheureusement conduites in vitro, mettent en évidence une certaine spécificité de l'effet protecteur. Différentes espèces mycorhiziennes ont étó confrontées à différentes souches de champignons pathogènes (MARX, 1969; SASIKK, 1967) (tabl. 4 et 5). Certaines espèces manifestent une aptitude antagoniste, repérée par une inhibition de croissance plus ou moins prononcée vis-à-vis de plu. sieurs espèces d'agents pathogènes. Suillus luteus, Leucopaxillus cerealis on ceute capacité, ainsi que Tricholoma saponaceum qui, toutefois, ne serait pas mycorhizogène. La situation la plus fréquente est un effet spécifique. L'extrapolation in vivo de ces résultats obtenus in vitro est très hasardeuse et mérite une confirmation par l'étude des mêmes espèces en conditions naturelles.

\subsection{Dépendance de l'aptitude prophylactique vis-à-1 is de l'environnement tellurique}

Cette dépendance est très marquée et deux études différentes do Davis (1979, 1980) le démontrent parfaitement. La première étude concerne le coton (Gossypiam 
hirsutum L.) confronté à un parasite vasculaire Verticillium dahliae. L'incidence du parasite est équivalente que la plante soit ou non mycorhizée avec Glomus fasci. culatus, lorsque le taux de phosphore atteint des valeurs élevées $(300 \mathrm{~kg} / \mathrm{g}$ ) (tabl. 6 et 7). L'endomycorhization conduit à une aggravation de la maladie. lorsque le taux de phosphore est bas $(20 \mathrm{~kg} / \mathrm{g})$.

\section{TABLEAU 4}

Influence de différentes espèces mycorhizogènes sur quelques agents pathogènes, in vitro.

D'après MarX (1969).

In vitro, influence of various mycorrhizal fungi on some plants pathogens.

\begin{tabular}{|c|c|c|c|c|c|}
\hline Agent pathogène & $\begin{array}{l}\text { Laccaria } \\
\text { laccata }\end{array}$ & $\begin{array}{l}\text { Lactarius } \\
\text { deliciosus }\end{array}$ & $\begin{array}{c}\text { Leucopaxillus } \\
\text { cerealis var. } \\
\text { piceana }\end{array}$ & $\begin{array}{l}\text { Pisolithus } \\
\text { tinctorius }\end{array}$ & $\begin{array}{l}\text { Suillus } \\
\text { luteus }\end{array}$ \\
\hline Fomes ammosus ..... & 0 & 0 & M & 0 & 0 \\
\hline Fusarium oxysporum & (9) & () & 0 & 0 & M \\
\hline $\begin{array}{l}\text { Phytophthora } \\
\quad \text { cinnamomi .... }\end{array}$ & 0 & $\mathrm{f}$ & TF & 0 & 1 \\
\hline $\begin{array}{l}\text { Pythium aphanider } \\
\quad \text { matum .......... }\end{array}$ & f & ( & $\mathbf{M}$ & 0 & $\mathrm{~F}$ \\
\hline Pythium ultimum .... & 0 & () & $\mathrm{F}$ & 0 & $\mathbf{M}$ \\
\hline Rhizoctonia solani . . & 0 & 0 & 0 & 0 & 0 \\
\hline
\end{tabular}

Tableau 5

Influence de champignons mycorhizogènes sur les agents de fonte des semis de Pinus spp., in vitro.

D’après SAsek (1967).

In vitro, influence of some mycorrhizal fungi on damping off agents of Pinus spp.

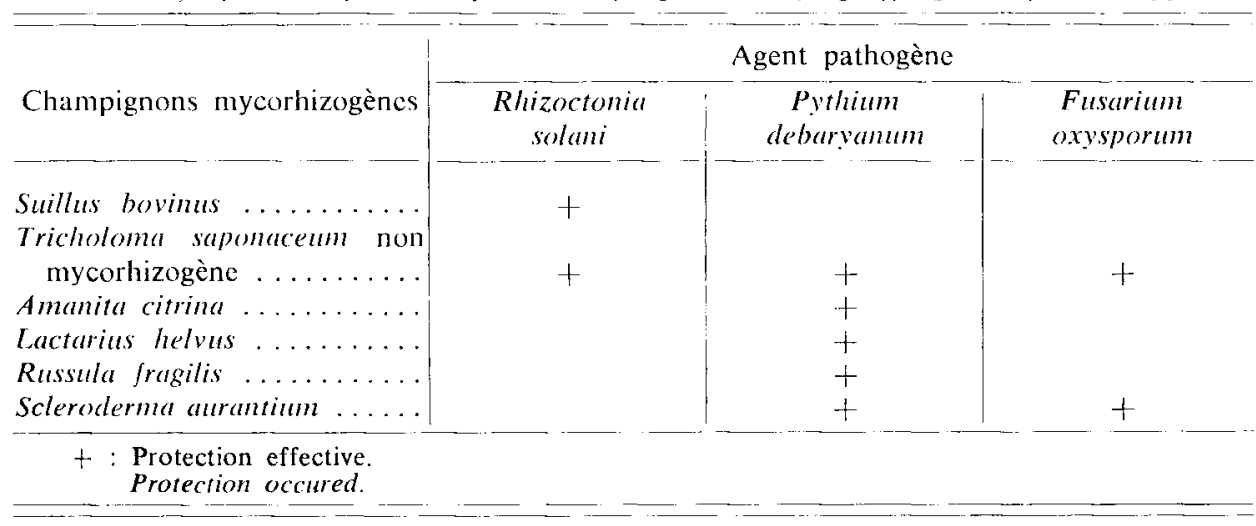


TABLEAU 6

Influence de la richesse en phosphore du sol sur les relations entre Verticillium dahliae et les mycorhizes formées par Glomus fasciculatus avec le coton (Gossypium hirsutum).

D'après Davis et al. (1979).

Effect of Glomus fasciculatus and two levels of phosphorus on the severity of verticillium wilt of cotton.

\begin{tabular}{|c|c|c|c|c|c|c|}
\hline \multirow{3}{*}{ Traitement } & \multicolumn{5}{|c|}{ Phosphore par $\mathrm{g}$ de sol } & \\
\hline & \multicolumn{3}{|c|}{$20 \mu \mathrm{g}$} & \multicolumn{3}{|c|}{$300 \mu g$} \\
\hline & $\begin{array}{c}\text { Poids sec } \\
\text { partic } \\
\text { aérienne }\end{array}$ & $\begin{array}{l}\text { Index de } \\
\text { coloration } \\
\text { vasculaire }\end{array}$ & $\begin{array}{c}\text { Propa- } \\
\text { gules/g } \\
\text { de pétiole }\end{array}$ & $\begin{array}{l}\text { Poids sec } \\
\text { partie } \\
\text { aérienne }\end{array}$ & $\begin{array}{l}\text { Index de } \\
\text { coloration } \\
\text { vasculaire }\end{array}$ & $\begin{array}{l}\text { Propa- } \\
\text { gules/g } \\
\text { de pétiolo }\end{array}$ \\
\hline & $(*)$ & & & & & \\
\hline Non infecté ... & $12,4 \mathrm{~b}$ & $0 \quad c$ & () b & 21,9 a & 0 & $0 \mathrm{~b}$ \\
\hline$V$. dahliae & $9,0 \mathrm{bc}$ & $18,5 \mathrm{~b}$ & $791 \mathrm{~b}$ & $4,0 \quad b$ & 79,3 a & $23575 a$ \\
\hline G. fasciculatus ... & 17.5 a & $0 \mathrm{c}$ & $0 \mathrm{~b}$ & 23.3 a & 0 & $0 \mathrm{~b}$ \\
\hline $\begin{array}{c}V . \text { dahliae }+G . \text { fasci- } \\
\text { culatus } \ldots \ldots \ldots\end{array}$ & $5,7 \mathrm{c}$ & 53,6 a & 11010 a & $5,1 \mathrm{~b}$ & 86,6 a & $21444 a$ \\
\hline
\end{tabular}

(*) A un indice différent correspond une différence significative au seuil de 5 p. 100.

Means followed by the same letter are not significantly different ( $p=0.05$ ) according to Duncan muliple range test.

\section{TABLEAU 7}

Influence de la richesse en phosphore du sol sur les relations entre Phytophthora parasitica et Citrus sinensis L. mycorhizé avec Glomus fasciculatus.

D'après Davis \& MENGE (1980).

Effect of Glomus fasciculatus and two levels of phosphorus on the severity of the disease caused by Phytophthora parasitica on Citrus sinensis.

\begin{tabular}{|c|c|c|c|c|c|c|}
\hline \multirow[b]{3}{*}{ Traitement } & \multicolumn{5}{|c|}{ Dose de phosphore $(\mu \mathrm{g} / \mathrm{g}$ sol) } & \multirow[b]{3}{*}{$\begin{array}{l}\% \text { de } \\
\text { racine } \\
\text { saine }\end{array}$} \\
\hline & \multirow{2}{*}{$\begin{array}{l}\text { Poids sec } \\
\text { partie } \\
\text { aérienne }\end{array}$} & \multicolumn{2}{|l|}{6} & \multicolumn{2}{|r|}{56} & \\
\hline & & $\begin{array}{l}\text { Poids sec } \\
\text { racine }\end{array}$ & $\begin{array}{c}\% \text { de } \\
\text { racine } \\
\text { saine }\end{array}$ & $\begin{array}{l}\text { Poids sec } \\
\text { partic } \\
\text { aćrienne }\end{array}$ & $\begin{array}{l}\text { Poids sec } \\
\text { racine }\end{array}$ & \\
\hline Non infecté . . & 0,74 & 0,37 & 100 & 1,99 & 0,61 & 100 \\
\hline P. parasitica & 0,36 & 0,14 & 33 & 1,14 & 0,47 & 74 \\
\hline G. fasciculatus & 3,50 & 1,04 & 100 & 4,40 & 1,37 & 100 \\
\hline $\begin{array}{l}\text { P. parasitica }+ \\
\text { G. fasciculatus. }\end{array}$ & 0,84 & 0,39 & 50 & 1,17 & 0,49 & 72 \\
\hline
\end{tabular}




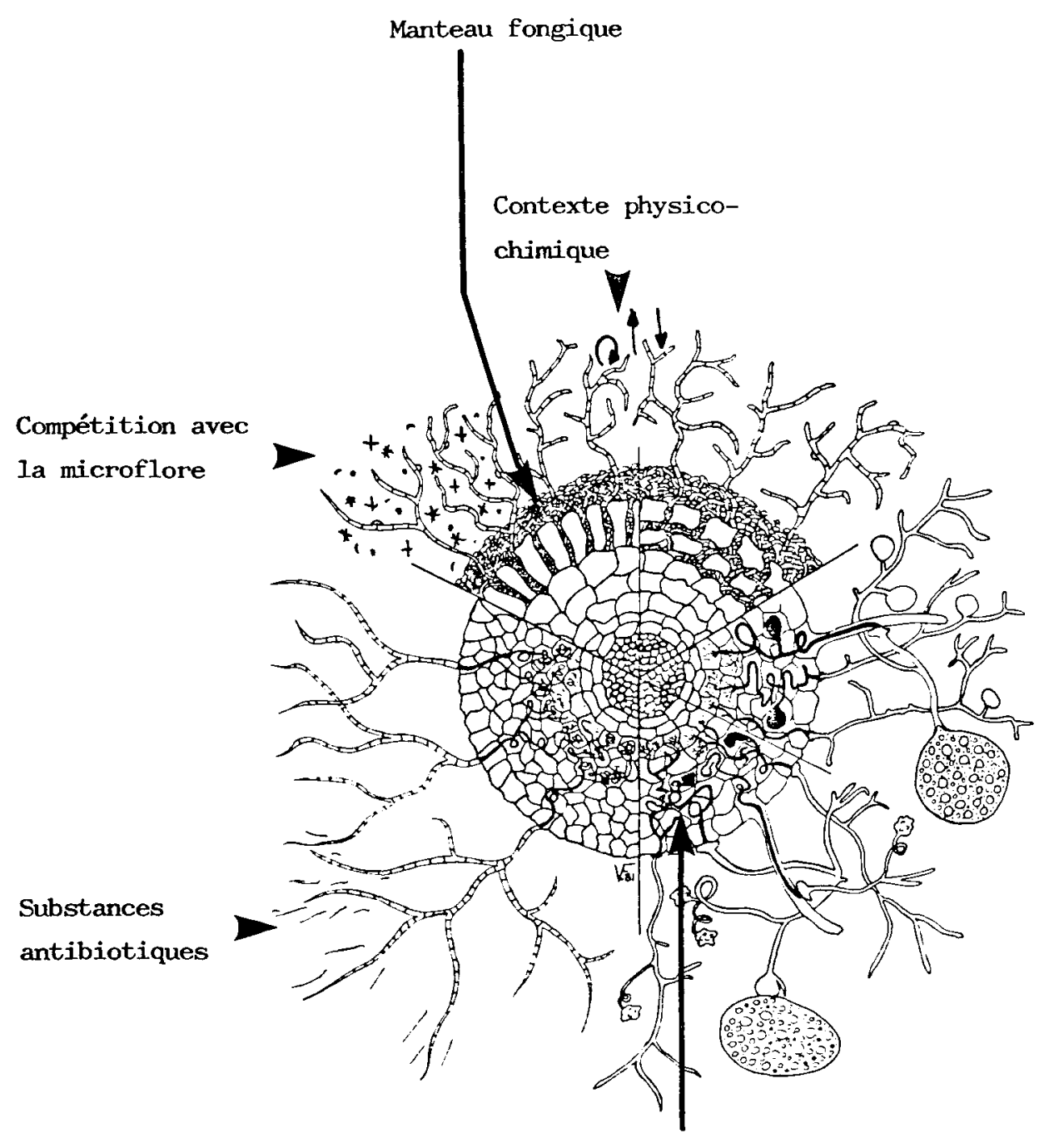

Substances inhibitrices

FIG. 2

Les différents mécanismes de résistance des racines mycorhizées, altx agents pathogènes telluriques.

(A partir d'un dessin de V. Furlan)

The different ways of the control of soil borne diseases by mycorrhizal roots.

(From Scheme made by V. FurLaN)

Ce schéma ne respecte pas le développement du mycélium relativement au diamètre racinaire. Le mycélium atteint en réalité 10 à 20 fois les dimensions figurécs sur ce dessin. Les différents mécanismes sont représentés indépendamment du type de mycorhize représentée.

Development of external hyphae is 10 to 20 more important than represented on this picture. Mechanisms are located on this scheme without relationship with the type of mycorrhizae. 
Une expérience analogue met en présence Citrus sinensis et Phytophthora parasitica agent de pourritures des racines. La maladie n'est pas modifiéc par l'endomycorhization avec $G$. fasciculatus lorsque le sol est riche en phosphore. Au contraire l'endomycorhization améliore sensiblement l'état sanitaire des plants élevés en sol pauvre en phosphore.

Le fonctionnement de la mycorhize est évidemment très différent selon le taux de phosphore du sol, et ces deux études démontrent que l'aptitude prophylactique en dépend directement.

A travers les quelques exemples précédents apparaît une particularité fondamentale de l'aptitude prophylactique des mycorhizes : c'est un potentiel qui s'exprimera plus ou moins complètement en fonction de l'environnement de la mycorhize. Si certaines exigences écologiques de quelques espèces mycorhizogènes sont bien délimitées (ex. : caractère thermophile de Pisolithus inctorius) on ignore tout ou presque sur les circonstances favorables à l'expression de l'aptitude prophylactique de la mycorhize. Les connaissances sont encore insuffisantes en ce domaine pour se prononcer sur l'intérêt et l'utilité de ce phénomène pour la lutte intégrée contre les agents pathogènes. A quoi bon sélectionner une souche mycorhizogène si son effet dissuasif ne s'exerce qu'en conditions particulières, exceptionnelles en conditions naturelles?

\section{Les différents mécanismes qui contribuent à la protection contre les agents pathogènes (fig. 2)}

ZaK (1969) puis MARX (1972) ont proposé une liste des différents mécanismes mis en jeu par les mycorhizes pour la protection du système racinairc des plantes.

Pour atteindre la racine, tout agent pathogène doit déjouer, successivement ou simultanément les différents systèmes de défense intervenant «à distance », donc participant à la création de l' «ambiance mycorhizosphérique " sous l'influence de là mycorhize.

Trois processus différents peuvent être distingués : l'agent pathogène doit évoluer dans un contexte physico-chimique transformé par l'activité de la mycorhize (prélèvements nutritifs ; alimentation hydrique ; modification des exsudats racinaires; sous-produit du métabolisme des partenaires ou de l'activité du champignon mycorhizogène; RambELLr, 1973). D'ailleurs une des conséquences les plus évidentes de la symbiose est d'étendre et de modifier considérablement cet espace transformé sur le plan physico-chimique.

L'agent pathogène doit entrer en compétition avec une microflore et une microfaune "contrôlées» par la mycorhize, adaptées à l'ambiance mycorhizosphérique, en interaction constante avec le milieu. L'intense activité microbienne qui règne à proximité de la racine fait de la mycorhizosphère le lieu des compétitions les plus intenses. STACK \& Sinclair (1973) sont les seuls à rapporter une influence d'un champignon mycorhizogène, Laccaria laccata avant son association avec la racine, sur un champignon pathogène, Fusarium oxysporum, par l'intermédiaire de la microflore. 
De nombreux travaux attestent de l'importance des modifications dans la composition et l'activité de la microflore liées à l'association mycorhizienne. L'étude de KATZNELSON et al. (1962) situe l'étendue des remaniements opérés par la symbiose dans la rhizosphère du bouleau jaune (Betula alleghaniensis) (tabl. 8). Les auteurs constatent une augmentation sensible du nombre de bactéries et d'actinomycètes et par contre une légère réduction du nombre de champignons sur la surface des racines mycorhizées. Parmi les bactéries, celles nécessitant des facteurs nutritifs complexes, sont en plus grand nombre sur la racine mycorhizée. La composition des populations fongiques varie considérablement. Des espèces de Pythium spp., Fusarium spp. et Cylindrocarpon spp. prédominent sur les racines non mycorhizées; les deux premières manquent totalement sur la racine mycorhizée où abondent des Penicillium spp., Paecilomyces, Phialocephala...

\section{TABLEAU 8}

Microflore associée aux racines mycorhizées et non mycorhizées du bouleau jaune. D'après KATZNELSON et al. (1962).

Microflora of mycorrhizal and non-mycorrhizal roots of yellow birch.

Flore totale.

\begin{tabular}{|c|c|c|}
\hline Microorganismes & Racine mycorhizée & Racine non mycorhizée \\
\hline Bactéries ... & 3930000 & 2650000 \\
\hline Actinomycètes $\ldots \ldots \ldots \ldots \ldots$ & 3000 & 340 \\
\hline Champignons . . . . . . . . . & 16 & 32 \\
\hline
\end{tabular}

En millier par $\mathrm{g}$ de racine sèche.

1000 par $g$ of died root.

Flore fongique, transcription partielle.

Partial transcription of fungic flora.

\begin{tabular}{|c|c|c|}
\hline \multirow{2}{*}{ Genre } & \multicolumn{2}{|c|}{ Pourcentage } \\
\hline & Racine mycorhizée & Racine non mycorhizée \\
\hline Pythium & 0 & 14 \\
\hline Mortierella & 3 & 0 \\
\hline Penicillium .............. & 16 & 0 \\
\hline Gliocladitm $\ldots \ldots \ldots \ldots \ldots$ & 2 & 0 \\
\hline Hyalostachybotris $\ldots \ldots \ldots \ldots$ & 0 & 8 \\
\hline Phialocephala $\ldots \ldots \ldots \ldots \ldots$ & 7 & 0 \\
\hline Fusarium $\ldots \ldots \ldots \ldots \ldots \ldots$ & 0 & 24 \\
\hline Cylindrocarpon $\ldots \ldots \ldots \ldots$ & 21 & 38 \\
\hline Paecilomyces $\ldots$ & 8 & 0 \\
\hline Total $\ldots \ldots \ldots \ldots \ldots \ldots \ldots$ & 100 & 91 \\
\hline
\end{tabular}


D'autres travaux, FoSTER \& MARKS (1967) révèlent qu'à différents types morphologiques de mycorhizes sont associées des microflores différentes.

A une telle disparité dans la composition de la microflore peuvent correspondre des répercussions écologiques très importantes notamment vis-à-vis des agents pathogènes. L'étude de KATZNELSON montre d'ailleurs que la symbiose mycorhizienne exclut tout agent pathogène de la racine mycorhizée du bouleau jaune. De même l'association du hêtre (Fagus sylvatica L.) avec Hebeloma crustuliniforme aboutit à la réduction du potentiel infecticux du sol infesté par Pythium spp., tandis qu'un autre champignon mycorhizogène Paxillus involutus le fait au contraire augmenter (PIsRIN \& GARBAyE, 1982).

L'agent pathogène doit subir l'effet de substances antibiotiques ou antifongiques élaborées par le champignon mycorhizogène, et libérées dans le sol. Un grand nombre d'espèces mycorhizogènes sont capables de produire de telles substances in vitro. In situ, la démonstration de ce phénomène est plus difficile. MarX \& Daver (1969) ont établi que Leucopaxillus cerealis var. piceana (Pecti.) produit deux antibiotiques : le diatretyne nitryle et le diatretyne 3 au niveau de la mycorhize et de la rhizosphère. Ces substances assurent même la protection de racines non mycorhizées directement adjacentes aux racines mycorhizées (tabl. 2). Suillus luteus est également à l'origine d'un effet d'antibiose cependant moins marqué.

A cet effet «à distance ", s'ajoutent des mécanismes intervenant au niveau de la racine :

- le manchon mycélien (manteau des ectomycorhizes) constituc un obstacle, une barrière mécanique difficile à franchir pour certains agents pathogènes. L'exemple le plus démonstratif est fourni par Marx (1969) (tableau 9). Les mycorhizes de Pinus taeda (inoculées) ou de Pinus echinata (naturelles) formant un manteau complet et un réseau de Hartig très dense, assurent une protection totale, de la racine à l'encontre de $P$. cinnamomi, tandis qu'un pourcentage élevé de mycorhizes ne constituant qu'un manteau et un réseau de Hartig très partiels sont infectés par le parasite;

- l'agent pathogène est confronté à des substances inhibitrices produites par l'hôte à la suite de l'infection mycorhizienne.

Dès les premières tentatives de pénétration d'un champignon mycorhizogène ou d'un agent pathogène, un processus de défense se déclenche chez la plante et se traduit par la production de substances appartenant aux phytoalexines.

MORANDI \& BAILEY (1984) ont montré que l'association endomycorhizienne chez. le soja (Glycine max.) exacerbe la synthèse de trois isoflavonoïdes : glyceolline I, coumestrol, et daidzéine. La concentration de ces substances dans les tissus en particulier le coumestrol dépasse les concentrations qui, in vitro, inhibent la mobilité d'un nématode phytophage (Pratylenchus spp.). A cette augmentation notable du taux de ces substances inhibitrices dans les tissus s'ajoute le fait que leur accumulation préexiste à l'agression des agents pathogènes.

Chez les résineux, KRUPA et al. (1973) rapportent que la concentration de substances appartenant au groupe des terpènes est multipliée par 30 à 40 lorsque les racines de Pinus echinata sont mycorhizées avec Pisolithus tinctorius ou Cenococcum graniforme. Ces substances sont volatiles et réduisent la croissance végétative d'un grand nombre de champignons pathogènes (tabl. 10). 


\section{Tableau 9}

Influence de la structure du manchon fongique des mycorhizes de Pinus taeda (a) et Pinus echinata (b) sur la pénétration des racines par Phytophthora cinnamomi.

D'après MARX (1969).

Influence of structure's mantle of the mycorrhizae of Pinus taeda (a) and Pinus echinata (b) on root penetration by Phytophthora cinnamomi.

(a)

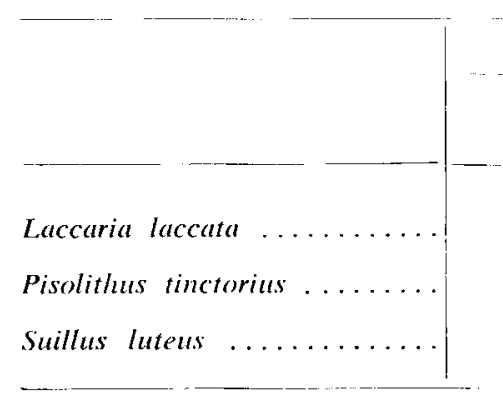

(b)

\begin{tabular}{|c|c|c|c|c|}
\hline \multirow{4}{*}{ Type racinaire } & \multicolumn{4}{|c|}{ Racines mycorhizées } \\
\hline & \multicolumn{2}{|c|}{3 jours après inoculation } & \multicolumn{2}{|c|}{10 jours après inoculation } \\
\hline & \multicolumn{4}{|c|}{ Inoculation avec $P$. cinnamoni } \\
\hline & Nbre inoculées & $\%$ infection & Nbre inoculées & $\%$ infection \\
\hline \multicolumn{5}{|l|}{ Mycorhize } \\
\hline Forme $1 \ldots \ldots \ldots$ & 12 & $25(*)$ & 14 & $43(*)$ \\
\hline Forme $2 \ldots \ldots$ & 17 & 0 & 7 & 0 \\
\hline Forme $3 \ldots \ldots \ldots$ & 23 & 0 & 11 & 6 \\
\hline $\begin{array}{l}\text { Racine courte non my- } \\
\text { corhizée } \ldots \ldots \ldots\end{array}$ & 16 & 100 & 11 & 100 \\
\hline $\begin{array}{c}\text { Racine latérale sans } \\
\text { mycorhize } \ldots \ldots \ldots\end{array}$ & 6 & 100 & 6 & 100 \\
\hline
\end{tabular}

(*) Mycorhize dont le manteau et le réseau de Hartig sont incomplètement développés. 


\section{Tableau 10}

Influence de l'association mycorhizienne sur la production de monoterpènes dans les racines de Pinus echinata.

(Les valeurs sont exprimées en $\mathrm{mg} / \mathrm{g}$ de poids sec,

l'augmentation de la production est exprimée par les rapports $B / A, C / A$ et $E / D$ ).

D'après KRUPA et al. (1973).

Influence of the mycorrhization on monoterpene production by the root of Pinus echinata.

(Data are given in $\mathrm{mg} / \mathrm{g}$ dry weight;

the increase of the production is expressed by the rates $B / A, C / A$ and $E / D)$.

\begin{tabular}{|c|c|c|c|c|c|c|}
\hline Monoterpène & a Pinène & $\beta$ Pinène & Myrcène & 3 - Carène & Limonène & $\begin{array}{l}\text { Phellan- } \\
\text { drène }\end{array}$ \\
\hline Racine courte A N.M. & 1,15 & 0,36 & 0,02 & 0,01 & 0,14 & 0,02 \\
\hline$P$. tinctorius $\mathrm{B} \quad \ldots$ & 2,88 & 0,52 & 0,06 & 0,43 & 0,23 & 0,03 \\
\hline $\mathrm{B} / \mathrm{A}$ & 2,5 & 1,4 & 3 & 43 & 1,64 & 1,60 \\
\hline $\begin{array}{l}\text { Cenococcum granifor- } \\
\text { me } C \ldots \ldots \ldots \ldots\end{array}$ & 1,60 & 0,23 & 0,04 & 0,01 & 0,05 & 0,60 \\
\hline$\ldots \ldots \ldots \ldots \ldots$ & 1,4 & 0,64 & 2 & 1 & 0,36 & 30 \\
\hline Racine courte D N.M. & 2,70 & - & - & 2,90 & 0,005 & 0,05 \\
\hline Boletus variegatus $\mathrm{E}$.. & 7,80 & 一 & - & 12,00 & 0,02 & 0,18 \\
\hline $\mathrm{E} / \mathrm{D}$ & 2,9 & 一 & - & 4,1 & 4,0 & 3,6 \\
\hline
\end{tabular}

N.M. = non mycorhizée - no mycorrhizae.

\section{Conclusion}

A la lucur de nos connaissances actuelles une conclusion s'impose : les mycorhizes possèdent incontestablement la capacité de protéger les plantes contre les agents pathogènes d'origine tellurique. Cette protection s'opère à travers des mécanismes variés, complémentaires qui agissent généralement de concert. Mais l'existence et l'efficience de ces différents processus connaissent des variations notables selon la plante, le champignon mycorhizogène, l'agent pathogène... De plus l'expression de cette protection est subordonnée au conditions de l'environnement sol où a lieu la confrontation mycorhize-agent pathogène. Les connaissances des facteurs qui modulent le fonctionnement des différents mécanismes font souvent défaut. Des questions essentielles demeurent sans réponses. Dans quelles conditions l'aptitude prophylactique des mycorhizes contribue-t-elle à réduire la gravité des maladies d'origine tellurique? Quelles sont les influences des pratiques culturales sur ce phénomène? Doit-on prendre en considération cette capacité de protection comme 
critère de sélection des souches mycorhizogènes? Ces énormes lacunes dans nos connaissances interdisent pour l'instant de se prononcer sur l'intérêt pratique de la maîtrise de ce phénomène.

Les mycorhizes, partie intégrante de la plante, offrent un potentiel naturel de protection contre les aggressions parasitaires qui peut sans doute parfaitement compléter et s'intégrer à d'autres interventions visant à minimiser les attaques parasitaires. D'ailleurs en pépinière où les pertes consécutives à diverses maladies dépassent fréquemment le bénéfice espéré d'une meilleure croissance, la valorisation de l'aptitude prophylactique des mycorhizes sera recherchée en priorité.

$\mathrm{Ni}$ panacée, ni chimère, trop longtemps ignorée, la capacité offerte aux plantes par les mycorhizes pour lutter contre les agents pathogènes est un potentiel de lutte biologique, qu’il nous faut apprendre à connaître, pour tenter de le maîtriser et de l'exploiter.

Reçu en seplembre 1984.

Accepté en mars 1985.

\section{Summary \\ The ability of mvcorrhizae to protect plants against diseases : panacea or chimera?}

The role of mycorrhizae as biological deterrents to diseases of plants are reviewed from the literature and author's experiments. The examples cited revealed that mycorrhizal association can act either as a source of improvement or aggravation of sanitary state of the plants.

The protective ability was only demonstrated for certain soil borne discases. The expression of this natural potential was related to several factors like nature of the hosts plants, mycorrhizal symbiots, plant pathogens, and conditions of the telluric environnement.

The practical exploitation of this phenomenon requires more in depth studies about the favourable conditions for expressing the protective ability of symbiotic associations.

\section{Références bibliographiques}

Atilano R.A., Rich J.R., Ferris M., Menge J.A., 1976. Effect of Meloidogyne arenaria on endomycorrhizal grape (Vitis vinefera) rootings. J. Nematol., 8, 278.

Bartschi H., Gianinazzi Piarson V., Vegil I., 1981. Vesicular-arbuscular mycorrhiza and root rot disease (Phytophthora cinnamomi Rands) development in Chamalecyparis lawsoniana (Murr.) Parl. Phytopathol. Z., 102, 213-218.

CORTE: A., 1969. Research on the influence of the mycorrhizal infection on the growth, vigor, and state of health of three Pinus species. Arch. Bot. Biogegr. Ital., 45, 1.

Davis R.M., Menge J.A., Erwin D.C., 1979. Influence of Glomus fasciculatus and soil phosphorus on Verficillitum wilt of cotton. Phytopath., 69, 453-456.

Davis R.M., MENGE J.A., 1980. Influence of Glomus fasciculatus and soil phosphorus on Phytophthora root rot of citrus. Phytopath., 70, 447-452.

DEHNE H.W., 1982. Interaction between vesicular arbuscular mycorrhizal fungi and plant pathogens. Phytopath., 72, 1115-1119. 
Foster R.C., Marks G.C., 1967. Observations on the mycorrhizae of forest trees. II - The rhizosphere of Pinus radiata D. Don. Aust. J. Biol. Sci., 20, 915.

Frank A.B., 1885. Über die auf Wurzelsymbiose beruhende Ernährung gewisser Baüme durch unterirdische Pilze. Ber. Deut. Bot. Ges., 3, 128.

Gianinazzj S., Gianinazzi Pearson V., Trouvelot A., 1982. Les mycorhizes, partie intégrante de la plante : biologie et perspectives d'utilisation. Les Colloques de l'I.N.R.A. Numéro 13, 397 p. I.N.R.A. éditeur.

Katznelson A., Rouatt B.N., Piteresson F.A., 1962. The rhizosphere effect of myeorrhizal roots of yellow birch seedlings. Can. J. Bot., 40, 77-382.

Krupa S., Andersson J., Marx D.H., 1973. Studies on ectomycorrhizac of pine. IV - Volatile organic compounds in mycorrhizal and nonmycorrhizal root systems of Pinus echinata mill. Eur. J. For. Path., 3, 194-200.

Marais L.J., Kotze P.M., 1976. Ectomycorrhizae of Pinus patula as biological deterrents to Phytophthora cinnamomi. S. Afric. For. J., 99, 35-39.

Marx D.H., 1969. Mycorrhizae : I - Ectomycorrhizac as biological deterrents to pathogenic root infections. Proceedings of the first NACOM, $n^{\circ 1189}$, USDA Forest Service, 81-96.

Marx D.H., Davey C.B., 1969. The influence of ectotrophic mycorrhizal fungi on the resistance of pine roots to pathogenic infections. III - Resistance of aseptically formed mycorrhizac to infection by Phytophthora cinnamomi, 59, 549-558.

MARX D.H., 1972. Ectomycorrhizae as biological deterrents to pathogenic root infections. Ann. Rev. Phytopath., 10, 429-454.

MORANDi D., Balley J.A., 1984. Isoflavonoid accumulation in soybean roots infected by vesicular arbuscular mycorrhizal fungi. Physiol. Plant Pathol., 24, 357-364.

Perrin R., Garbaye J., 1982. Influence of ectomycorrhizae on infectivity of Pythium infested soils and substrates. Plant and Soil, 71, 345-351.

Rambelli A., 1973. The rhizosphere of mycorrhizae. In Ectomycorrhizae. G.C. Marks and T.T. Koslowski editors. Academic Press. New York, 299-349.

Ross J.P., 1972. Influence of Endogone mycorrhizac on Phytophthora root rot of soybean. Phytopath., 62, 896-897.

SASEK V., 1967. The protective effect of mycorrhizal fungi on the host plant. 14th IUFRO Congress, Münich, 24, 182-190.

SCHenCK N.C., Ridings W.H., Cornell J.A., 1977. Interaction of two vesicular arbuscular mycorrhizal fungi and Phytophthora parasitica on two citrus root stocks. Abstr., 9, Proc. Third NACOM, Corvallis, Oregon.

SCHOENBECK F., 1980. Endomycorrhiza : Ecology, function and phytopathological aspects, Forum Microbiol., 3, 90-96.

SiKora R.A., SCHOENBEck F., 1975. Effect of vesicular arbuscular mycorrhizae (Endogone mosseae) on the population dynamics of the root knot nematodes (Mcloidogyne incognita and Meloidogyne hapla). VIII - Int. Congr. Plant Protect. J., 5, 158-166.

Sinclair W.A., Cowles D.P., HeE S.M., 1975. Fusarium root rot of Douglas fir seedlings : suppression by soil fumigation, fertility management, and inoculation with spores of the fungal symbiont Laccaria laccata. For. Sci., 21, 390-399.

Stack R.W., Sinclair W.A., 1975. Protection of Douglas fir seedlings against Fusurium root rot by a mycorrhizal fungus in the absence of mycorrhiza formation. Phytopath., $65,468-472$.

Woodhead S.H., Gerdemann J.W., Paxton J.D., 1977, Mycorrhizal infection of soybean roots reduces Phytophthora root rot (Abstract) 10, Third NACOM Proc.

ZaK B., 1964. Role of mycorrhizae in root disease. Ann. Rev. Phytopath., 2, 377. 